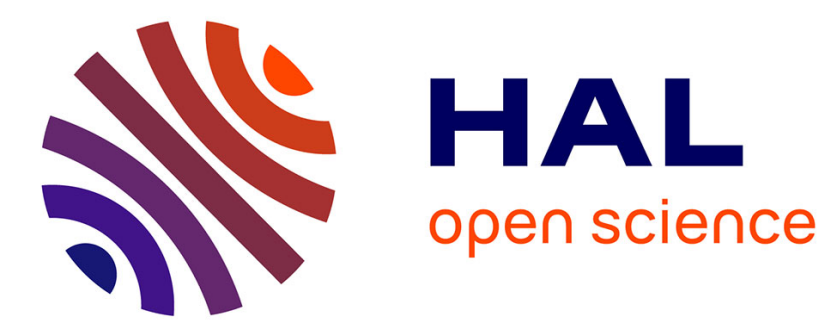

\title{
Estimation of Illuminants From Projections on the Planckian Locus
}

Baptiste Mazin, Julie Delon, Yann Gousseau

\section{To cite this version:}

Baptiste Mazin, Julie Delon, Yann Gousseau. Estimation of Illuminants From Projections on the Planckian Locus. IEEE Transactions on Image Processing, 2015, 24 (6), pp. 1944 - 1955. 10.1109/TIP.2015.2405414 . hal-00915853v3

\section{HAL Id: hal-00915853 \\ https://hal.science/hal-00915853v3}

Submitted on 9 Oct 2014

HAL is a multi-disciplinary open access archive for the deposit and dissemination of scientific research documents, whether they are published or not. The documents may come from teaching and research institutions in France or abroad, or from public or private research centers.
L'archive ouverte pluridisciplinaire HAL, est destinée au dépôt et à la diffusion de documents scientifiques de niveau recherche, publiés ou non, émanant des établissements d'enseignement et de recherche français ou étrangers, des laboratoires publics ou privés. 


\title{
Estimation of Illuminants From Projections on the Planckian Locus
}

\author{
Baptiste Mazin, Julie Delon and Yann Gousseau
}

\begin{abstract}
This paper introduces a new approach for the automatic estimation of illuminants in a digital color image. The method relies on two assumptions. First, the image is supposed to contain at least a small set of achromatic pixels. The second assumption is physical and concerns the set of possible illuminants, assumed to be well approximated by black body radiators. The proposed scheme is based on a projection of selected pixels on the Planckian locus in a well chosen chromaticity space, followed by a voting procedure yielding the estimation of the illuminant. This approach is very simple and learning-free. The voting procedure can be extended for the detection of multiple illuminants when necessary. Experiments on various databases show that the performances of this approach are similar to those of the best learning-based state of the art algorithms.
\end{abstract}

\section{INTRODUCTION}

The chromaticities of objects in a scene are highly dependent on the light sources. Humans have the ability, known as color constancy, to perceive objects almost independently of the illuminants. In contrast, the influence of illuminants may be a limiting factor for computer vision applications such as object recognition or categorization. The goal of automatic color constancy is to reduce these color variations as much as possible, and to render colors regardless of the light source, mimicking in a way the human capacity. The ability to produce image representations as independent as possible of the illuminants is also necessary for computational photography, where this process is often referred to as white balance. The first step of automatic color constancy consists, for a given scene, in estimating the colors of the illuminants. In a second step, when dealing with a single illuminant, the image can be globally corrected using this estimate so that it appears as if taken under a canonical illuminant. In this paper, we focus on the illuminant estimation step. We take interest in situations where one (single illuminant case) or several (multiilluminant case) light sources are involved in the scene.

\section{A. Single illuminant estimation}

Illuminant estimation has been a very active research field in the past decades, see for instance the recent survey [27]. In the case of a single light source, the simpler methods rely only on the distribution of colors in images. For example, a very popular way to estimate illuminant chromaticities in images is to assume that under a canonic light, the average RGB value observed in a scene is grey. This assumption gives rise to the Grey-World algorithm [7], which consists in computing the average color in the image and compensating for the deviation due to the illuminant. Several refinements have been proposed, restraining the hypothesis to well chosen surfaces of the scene, that are assumed to be grey [41]. Let us mention the work [54], which makes use of an invariant color coordinate [20] that depends only on surface reflectance and not on the scene illuminant. Unfortunately, thresholding this coordinate is generally not enough to select grey pixels correctly. An extension of this work [55] adds a clustering step to refine the selection. Potentially grey pixels can also be selected as the points close to the Planckian locus 11 in a given chromaticity space [39]. This approach may fail when two potentially grey surfaces are present in the scene.

Alternatively, the White-Patch assumption [40] supposes that a surface with perfect reflectance is present in the scene and that this surface correspond to the brighter points in the image. This results in the well-known Max-RGB algorithm, which infers the illuminant by computing separately the maxima of the RGB channels. Variants propose to perform a preliminary filtering of the image [1], or to replace the maximum by an average value on a small percentage of the brighter pixels [9]. A unified framework has been proposed for these two assumptions (Grey-World and White-Patch) in [24]. More recently, the same unified framework has been extended to the reflectance first and second order derivatives in the socalled Grey-Edge methods [50], [30], [8].

Another way to estimate the chromaticity of light sources is to rely on the dichromatic reflectance model [15], which takes into account the specular part of the reflectance at each pixel. Under this model, the pixels of a monochromatic surface belong to a plane in the RGB color space of the camera, or to a line in a well chosen chromaticity space, see [23]. Finding the intersection of those planes permits to recover the illuminant. In the case of an image containing only one monochromatic object, the illuminant can still be recovered by assuming that the light source chromaticity belongs to the Planckian locus.

Physical constraints on the set of illuminants and on the scene content are also used by Sapiro in [47], where it is assumed that reflectance spectra and illuminant spectral power distributions can be approximated by linear combinations of a small number of known basis functions. A voting procedure is then proposed, where each pixel votes for a set of possible illuminants. The Hough transform permits to retrieve the most probable illuminant.

More involved techniques rely on a learning phase, where color statistics are learned on a training set of images, taken under known light sources. Among these methods, gamut-

\footnotetext{
${ }^{1}$ In a chromaticity space, the Planckian locus refers to the set of black body radiators chromaticities.
} 
based approaches rely on the idea that the range of observable colors under a given illuminant is limited [25]. These approaches require to learn the set of observable colors under a known illuminant and to find the best feasible mapping between the colors of the observed scene and this set. The chromaticity of the unknown illuminant is then deduced from this mapping. The gamut mapping problem remains nonetheless ill-posed, and additional hypotheses are generally necessary [17], [27]. For instance, these hypotheses may concern the diversity of colors in the observed scene [25], or the set of possible light sources [17]. In order to go further, some authors explore not only the range but also the distribution of colors under canonical illuminants [22], [45]. This permits to recast the illuminant estimation as a maximum likelihood problem [22]. Other methods also involve a simple learning of color statistics [26].

While they offer correct estimations in a large number of situations, all of the previously mentioned approaches present different failure cases. A last category of illuminant estimation techniques thus proposes to combine the output of several of these methods [51], [4], [32]. The optimal combination strategy is learned on training image datasets, eventually relying on the statistical properties of natural images, or on more involved semantic information. Recently, Vasquez-Corral et al. [52] propose to refine color constancy approaches by incorporating perceptual constraints, weighting the set of illuminants "according to their ability to map the corrected image onto specific colors", chosen as universal color categories.

\section{B. Multi illuminant estimation}

All of the previous methods assume that only one illuminant is present in the scene and that this illuminant is uniform. Less attention has been paid in the literature to the multi-illuminant case, or to the case of a varying illuminant, although they can be considered as more realistic in practice. Typical examples are the case of outdoor images, with objects illuminated by direct sunlight and objects in shadows receiving light scattered by the sky, or the case of indoor scenes with both an incandescent light source and a natural light source coming from a window.

The Retinex theory proposed by Land and McCann [40] is one of the first model addressing the problem of local color constancy. In order not to confuse changes in illumination from changes in reflectance, the method assumes that the illuminant varies spatially smoothly: small changes are thus interpreted as illumination variations, while large ones are supposed to be due to reflectance changes. Several papers draw on the same ideas and try to incorporate additional constraints to the problem, either on the set of possible illuminants [19], [2] (assumed to be on the Planckian locus) or on the set of surfaces reflectances [2]. These approaches [19], [2] also exploit the illumination variations along matte surfaces to constrain the solution 2 , The work of Finlayson [19] is extended in [38], by including noise in the model, and refined in [37] for outdoor

\footnotetext{
${ }^{2}$ These surfaces must first be identified, for instance thanks to a segmentation step [2].
}

scenes, relying on the differences between shadowed and nonshadowed regions.

In [14], Ebner proposes a very simple solution to the local illuminant estimation, using a local and iterative version of the grey world algorithm to compute an illuminant estimation at each pixel. This algorithm is refined in [16] by using a nonuniform averaging of local pixels to address sudden illuminant variations. More recently, the authors of [5] propose to apply different color constancy algorithms on super-pixels and to combine their results. In a similar way, Gijensij et al. [31] evaluate color constancy algorithm on image patches, sampled with different strategies.

Some authors uses specular highlights [35] to estimate the spectral power distribution of illuminants through a spectral imaging device.

Finally, different user guided methods have been proposed in the literature [34], [6]. The method described in [34] permits to estimate for each image pixel the mixture between two illuminants specified by the user. This approach works well when the transitions between illuminants are smooth but fails in case of spatially abrupt illuminant changes. To address this problem, Boyadzhiev [6] proposes an algorithm allowing to correct images based on user indications on the nature of the surfaces, specifying whether they are neutral or uniform.

\section{Contributions of the paper}

The goal of this paper is to introduce a simple and effective method for the estimation of light sources chromaticities, without relying on any learning phase. Our approach draws on a novel voting procedure, combined with two physical assumptions, one concerning the content of the observed scene and the other concerning the set of possible illuminants. First, it is assumed that the scene contains at least some achromatic (or perfectly reflective) pixels; second, the feasible illuminants chromaticities are supposed to be in the vicinity of the Planckian locus. A refined version of the proposed voting procedure permits to deal with situations where more than one illuminant is involved in the scene, and to automatically estimate the number of these illuminants. The whole algorithm does not require any learning step and uses intuitive parameters. The method being applied globally on the image, the result varies only slightly with the scales of images. Various experiments show the efficiency of the proposed method when compared to state of the art approaches on different databases. A conference proceedings version of the first part of this work was published in [42].

The paper is organized as follows. In Section II we recall the color formation model and discuss color constancy. Section III describes the main steps of our color constancy algorithm in the case where only one illuminant has to be estimated. The multi-illuminant version is detailed in Section IV In Sections $\mathrm{V}-\mathrm{B}$ and $\mathrm{V}-\mathrm{C}$, the ability of the algorithm to retrieve light sources chromaticities is tested on several databases, both in the mono-illuminant and multi-illuminant cases. 


\section{ILLUMINANTS AND COLORS IN IMAGES}

This section presents a brief reminder on color image formation and on color constancy. We also recall the concept of black-body radiator, which is used in the rest of the paper to constrain the set of possible illuminants.

\section{A. Color image formation}

Let us denote by $E(., \mathbf{m})$ the irradiance spectrum falling on the camera sensor at pixel $\mathbf{m}$ and by $\rho(\lambda)=$ $\left(\rho_{R}(\lambda), \rho_{G}(\lambda), \rho_{B}(\lambda)\right)$ the camera sensitivities functions. The vector $\mathbf{p}(\mathbf{m})=\left(p_{R}(\mathbf{m}), p_{G}(\mathbf{m}), p_{B}(\mathbf{m})\right)$ measured by the camera at $\mathbf{m}$ is given by

$$
p_{c}(\mathbf{m})=\int_{\Lambda} E(\lambda, \mathbf{m}) \rho_{c}(\lambda) d \lambda, \quad c \in\{R, G, B\}
$$

where $\Lambda$ is the visible spectrum. The different image formation models differ in the way they describe the spectrum $E(., \mathbf{m})$ reaching the camera sensor at pixel $\mathbf{m}$. One of the most popular descriptions is given by the dichromatic reflection model [48], which decomposes $E(., \mathbf{m})$ in two parts, a Lambertian component and a specular component:

$$
E(\lambda, \mathbf{m})=L(\lambda, \mathbf{m})\left(\alpha_{l}(\mathbf{m}) S(\lambda, \mathbf{m})+\alpha_{s}(\mathbf{m})\right),
$$

where $L(., \mathbf{m})$ is the illuminant spectrum at $\mathbf{m}, S(., \mathbf{m})$ is the object reflectance spectrum at $\mathbf{m}$ and where $\alpha_{l}(\mathbf{m})$ and $\alpha_{s}(\mathbf{m})$ weight the Lambertian and specular components. The simpler Lambertian model ignores the specular term.

The illuminant estimation problem is intrinsically underconstrained: even in the case of a single illuminant, uniform across the scene, it is not possible to recover the complete power distribution $L(\lambda)$ from the discrete sensor measures $p_{c}(\mathbf{m})$. In practice, color constancy only aims at computing for each given pixel $\mathbf{m}$ the triplet of values $\left(\int_{\Lambda} \rho_{c}(\lambda) L(\lambda, \mathbf{m}) d \lambda\right)_{c \in\{R, G, B\}}$. Estimating these values from the observations is still an ill-posed problem and generally necessitates strong assumptions on the image content or on the illuminant properties, as described in the following paragraphs.

\section{B. Color correction and the Von Kries diagonal model}

In practice, it is usual to assume that the illuminant spectrum $L$ is constant over the whole image and that the camera sensitivity functions can be approximated by Dirac delta functions 3 associated to wavelengths $\lambda_{R}, \lambda_{G}$ and $\lambda_{B}$. Under these hypotheses, Equation (1) can be rewritten as

$p_{c}(\mathbf{m})=L\left(\lambda_{c}\right)\left(\alpha_{l}(\mathbf{m}) S\left(\lambda_{c}, \mathbf{m}\right)+\alpha_{s}(\mathbf{m})\right), \quad c \in\{R, G, B\}$.

In this case, the illuminant estimation problem amounts to retrieve $\left(L_{R}, L_{G}, L_{B}\right):=\left(L\left(\lambda_{R}\right), L\left(\lambda_{G}\right), L\left(\lambda_{B}\right)\right)$. Assuming that this illuminant has been retrieved, computing a version of the same image taken under another illuminant $L^{0}$ amounts to

\footnotetext{
${ }^{3}$ This assumption, very common in the literature, is only an approximation Nonetheless, observe that a sharpening technique can be applied if the sensors response functions are not sufficiently narrow-band [18].
}

multiply the vector $\left(p_{R}(\mathbf{m}), p_{G}(\mathbf{m}), p_{B}(\mathbf{m})\right)$ by the diagonal matrix [44]

$$
\left(\begin{array}{ccc}
\frac{L_{R}^{0}}{L_{R}} & 0 & 0 \\
0 & \frac{L_{R}^{0}}{L_{R}} & 0 \\
0 & 0 & \frac{L_{R}^{0}}{L_{R}}
\end{array}\right) .
$$

\section{Distribution of feasible illuminants}

It is often necessary to impose a priori information on the chromaticity of feasible illuminants to solve the color constancy problem. A possible hypothesis in this context is to assume that the possible illuminants are well represented by black-body radiators. According to the Planck model, the spectrum of light emitted by such an idealized physical body only depends on its temperature $T$ and is given by

$$
L(T, \lambda)=c_{1} \lambda^{-5}\left[\exp \left(\frac{c_{2}}{\lambda T}\right)-1\right]^{-1},
$$

where $T$ is the temperature in kelvins, $\lambda$ is the wavelength and $c_{1}=3.74183 \times 10^{16} \mathrm{Wm}^{2}$ and $c_{2}=1.4388 \times 10^{-2} \mathrm{mK}$ are two constants.

Planck's formula is an accurate estimation of the spectrum of most natural illuminants including daylight [36]. Besides, even if artificial light sources (such as fluorescent lights) generally have a power spectrum which is not well represented by the Planck formula, it was shown that such sources produce a set of perceived colors which is very close to those produced by a black-body radiator. Indeed, as underlined in [21], "for almost all daylights and typical man-made lights, including fluorescents, there exists a black-body radiator (...) which (...) will induce very similar RGBs for most surface reflectances".

\section{Colorspaces and chromaticity spaces}

In order to make use of the black-body hypothesis on the set of feasible illuminants, we need to transfer the camera related measures $\mathbf{p}(\mathbf{m})=\left(p_{R}(\mathbf{m}), p_{G}(\mathbf{m}), p_{B}(\mathbf{m})\right)$ to a more standard colorspace, where the Planckian locus is known. In this paper, we rely on the standardized colorspace CIE 1931 XYZ [15]. The conversion from the RGB coordinates of the camera to $\mathrm{XYZ}$ is linear and device dependent. We assume in this paper that this conversion matrix is known (conversions matrices can be found in the source code of dcraw [11], for instance).

Working with a universal colorspace has other benefits. First, the algorithm parameters do not have to be adapted to the camera color space and can be defined once and for all. Second, the computation of color distances and the projections on the Planckian locus are also much more sound in universal color spaces than in camera-specific spaces.

Now, when solving the color constancy problem, it is not possible to distinguish which part of the observed intensity comes from the light source and which part comes from the object reflectance. For this reason, the intensity information at $\mathbf{m}$ cannot be recovered and the illuminant is only estimated up to a multiplicative factor. When trying to retrieve this illuminant, it is thus sensible to work in a chromaticity space, i.e. a $2 \mathrm{D}$ representation of the $3 \mathrm{D}$ color space discarding the 
intensity component. For instance, the $x y$ chromaticity space is obtained by normalizing the $X Y Z$ triplet into $(x, y)=$ $\left(\frac{X}{X+Y+Z}, \frac{Y}{X+Y+Z}\right)$.

In this paper, we also make use of the CIE 1960 uv chromaticity space, proposed by MacAdam, and mostly used today to define correlated color temperatures (CCT) ${ }^{4}$ According to the CIE, the isotherms (lines of constant CCT) are defined in this space as the lines perpendicular to the Planckian locus [53]. It follows that the correlated color temperature $T(\mathbf{m})$ of a pixel $\mathbf{m}$ is the one of its orthogonal projection on the Planckian locus, i.e the one of its nearest black body radiator. The conversion from XYZ to uv is composed of a linear transformation, followed by a normalization to discard the intensity component.

Figure 1 shows the curve of black body radiators chromaticities, called the Planckian locus, in the CIE $1960 u v$ chromaticity diagram (i.e. the set of visible colors in the $u v$ space). We also display on the same diagram the chromaticities of a large variety of illuminants (obtained from the Colorchecker database, described in [26]). Observe that all of these chromaticities lie on (or close to) the Planckian locus.

\section{VOTING FOR THE MOST PROBABLE ILLUMINANT}

In this section, we present a first version of our illuminant estimation algorithm. We assume in the following that a single illuminant is used. As described above, estimating the triplet $\left(L_{R}, L_{G}, L_{B}\right)$ from the measured triplets $\mathbf{p}(\mathbf{m})$ is an underconstrained problem and it necessitates some additional assumptions.

\section{A. Working hypotheses}

We rely here on two hypotheses.

The first one concerns the set of possible illuminants of a scene. We assume that this set is not far from the Planckian locus, which means that the illuminants are supposed to be well approximated by black-body radiators. This a priori is quite common in the literature and was shown to be a reasonable assumption for most illuminants [36], [21].

Our second assumption concerns the scene content and is inspired by the white-patch method: we assume that the scene contains some achromatic or perfectly reflective surfaces. While it is possible to find or create counter examples, this assumption is more often satisfied in practice than the one of the grey-world algorithm (the average reflectance of a scene is grey), especially for scenes containing man-made objects. Both hypotheses will be discussed in the section devoted to the algorithm evaluation.

\section{B. Algorithm description}

The main idea of our method is to select pixels close to the Planckian locus and to make these pixels vote for the most probable illuminant. Before describing the algorithm more precisely, let us recall that the conversion matrix between the RGB coordinates of the camera and the XYZ colorspace is

\footnotetext{
${ }^{4}$ The correlated color temperature of a light source is the temperature of the black body radiator with the most perceptually similar color.
}

assumed to be known and that $\mathrm{Y}$ is the luminance component in this space.

The first part of the algorithm works as follows. First, for all pixels $\mathbf{m}$, the observed color triplet $\mathbf{p}(\mathbf{m})$ is transformed in a $2 \mathrm{D}$ chromaticity vector $\mathbf{c}(\mathbf{m})=(u(\mathbf{m}), v(\mathbf{m}))$ in the CIE 1960 uv diagram. We also compute the luminance $Y(\mathbf{m})$ of the color triplet in the space XYZ. Next, all pixels at a distance from the Planckian locus $\mathcal{L}$ larger than a threshold $\delta$ in the uv diagram are discarded, which means that we only keep pixels m such that

$$
\min _{\mathbf{r} \in \mathcal{L}}\|\mathbf{c}(\mathbf{m})-\mathbf{r}\|_{2}<\delta
$$

these pixels being considered as the most probably grey in the image. We then project each of the remaining chromaticities $\mathbf{c}(\mathbf{m})$ orthogonally on the Planckian locus:

$$
\mathbf{c}_{\text {Planck }}(\mathbf{m}):=\operatorname{proj}_{\mathcal{L}}(\mathbf{c}(\mathbf{m}))=\arg \min _{\mathbf{r} \in \mathcal{L}}\|\mathbf{c}(\mathbf{m})-\mathbf{r}\|_{2} .
$$

If the argmin in the previous equation is not uniquely defined 5 we choose randomly one of the possible projections. This assignment permits to define the correlated color temperature $T(\mathbf{m})$ of the pixel $\mathbf{m}$, as the temperature of the black body radiator whose chromaticity is $\mathbf{c}_{\text {Planck }}(\mathbf{m})^{6}$. We keep only the pixels whose correlated temperature falls inside a given interval $\left[T_{\min }, T_{\max }\right]$, containing the temperatures of the most common illuminants.

The second step of the algorithm is a voting procedure permitting to retrieve the most probable temperature $T^{0}$ for the illuminant. At the end of the first step, the remaining points in the chromaticity diagram are used to build a temperature histogram. For computing this discrete histogram, we convert the correlated temperature $T$ (measured in Kelvin) into the MIRED (Micro Reciprocal Degree) scale $T_{\text {Mired }}=10^{6} / T$ before the discretization. Indeed, the Kelvin temperature scale does not yield a satisfying sampling of the Planckian locus. The conversion to the MIRED scale is advocated by Priest [43] as providing a better sampling of the color temperature according to human perception. When computing the temperature histogram, the contribution of each pixel $\mathbf{m}$ is weighted by a power of its luminance $Y(\mathbf{m})$. This weighting is a way to favor the brightest pixels in the image, and to limit the influence of the darkest ones without completely discarding them 7 . The temperature $T^{0}$ of the illuminant is then estimated as the most represented one in this weighted histogram. For the sake of simplicity, the argmax of the histogram is chosen here, but a more sophisticated procedure, based on mode detection, can also be applied. We will see in the next Section how such a procedure can be used for the detection and estimation of several illuminants.

At the last step of the algorithm, we compute the barycenter of all pixels $\mathbf{m}$ who have participated in the choice of $T^{0}$ (those pixels which were kept to build the histogram and

\footnotetext{
${ }^{5}$ If the chromaticity of a pixel is far from the Planckian locus, Equation 7 may have several solutions.

${ }^{6}$ In practice, we use the approximation proposed by [33] to compute the projections and correlated color temperatures.

${ }^{7}$ In a way, this weighting scheme can be seen as a trade off between the Grey-World and the White-Patch hypotheses [24].
} 
whose discrete correlated color temperature falls in the bin $\left.T^{0}\right)$. In other words, all the chromaticities remaining at this point are averaged to compute the final estimation. This barycenter, which may be outside of the Planckian locus but remains close to it, is chosen as the output illuminant. Algorithm 11 gives a complete description of the procedure.

input : Image $\mathbf{p}$ in RGB (camera colorspace). Thresholds $\delta, T_{\min }, T_{\max }$, number $N$ of bins in the histogram, power $n$, canonical illuminant $\left(u^{\mathrm{ref}}, v^{\mathrm{ref}}\right)$.

output: Chromaticity $\left(u^{e}, v^{e}\right)$ estimated in the CIE 1960 uv space.

Initialize the histogram $H=0$ on $N$ bins;

for each pixel $\mathbf{m}$ do

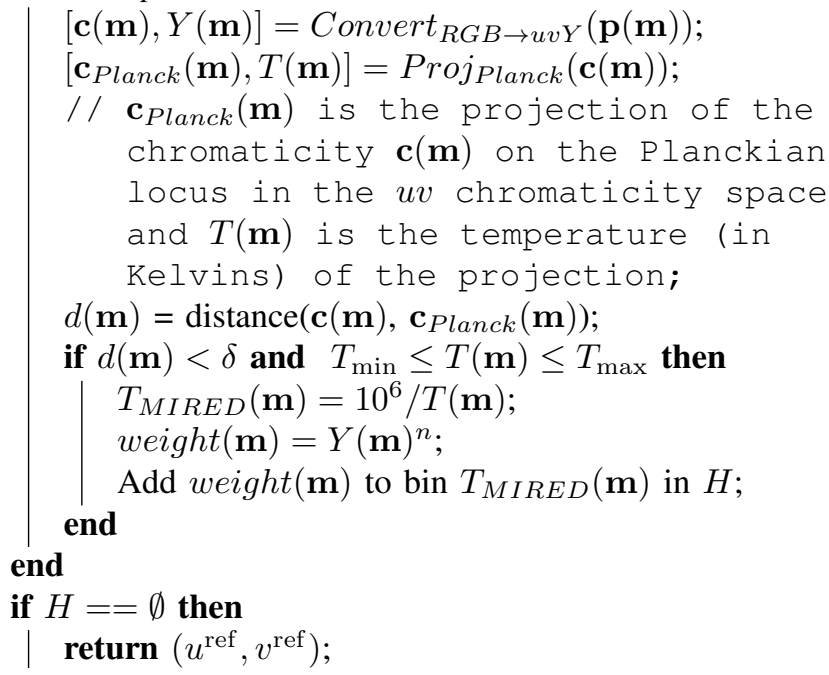

Algorithm 1: Illuminant selection algorithm. In practice, the following parameters provide good results on various dataset $\delta=0.0125, T_{\min }=2000 \mathrm{~K}, T_{\max }=20,000 \mathrm{~K}, \mathrm{~N}=30$, and $n=3$. These parameters are used for all experiments in Section $\mathrm{V}$.

\section{ALGORITHM REFINEMENT WITH MODES SELECTION}

The algorithm described in the previous section outputs a single illuminant after selecting the principal mode of the temperature histogram in a rough way (argmax of the histogram). In practice, the histogram may contain multiple modes, especially when several illuminants are present in the scene. Moreover, even with a single illuminant, the argmax choice is not always the most appropriate, since the mode may extend over a large range of temperatures. In this section, we propose a mode selection algorithm, inspired from a contrario methods [13], which permits to refine the detection and to manage the multi-illuminant cases.

Since we do not have any a priori information on the number of illuminants present in the scene, segmentation- based approaches such as k-Means cannot be used straightforwardly. Gaussian mixtures with a minimum description length criteria can be used to address the problem, but this would introduce an a priori on the shape of the modes that is not very accurate in practice. We propose here a generic algorithm which detect modes in an histogram. The algorithm is inspired from the a contrario mode selection presented in [13], [12]. It is adapted to the specific weighting procedure used to build the temperature histogram.

Let $H$ be an histogram with $N$ bins, each bin corresponding to a CCT interval in the mired scale. This histogram is obtained as explained in Algorithm 11: the contribution of each pixel $\mathbf{m}_{i}$ $i \in\{1, \ldots, M\}$ is weighted by a mass $w_{i}$ equal to a power function of its luminance. Following the general idea of $a$ contrario approaches [13], modes are detected as intervals in which the total mass contradicts a null hypothesis $\mathcal{H}_{0}$. Let us describe more precisely this hypothesis $\mathcal{H}_{0}$. For a given interval $[a, b]$ and a pixel $\mathbf{m}_{i}$ we define two random variables $B_{i}$ and $W_{i}$. The first one $B_{i}$, corresponds to the event "the CCT of $\mathbf{m}_{i}$ belongs to $[a, b]$ ". The second random variable $W_{i}$ corresponds to the mass $w_{i}$ of $\mathbf{m}_{i}$. The variables $B_{i}$ and $W_{i} i \in\{1, \ldots, M\}$ are said to follow the null hypothesis $\mathcal{H}_{0}$ if they are mutually independent, if all variables $B_{i}$ follow the same Bernoulli law of parameter $p_{[a, b]}\left(p_{[a, b]}\right.$ being the relative length of the interval $[a, b]$ in the histogram) and if all the variables $W_{i}$ follow an exponential law with expectation $\lambda 8$. The exponential distribution have been chosen because it is a reasonable approximation of the law of weights $w_{i}$ on most natural images. In practice, the parameter $\lambda$ is learned for each image. Now, let us consider the random variable $D_{[a, b]}=\sum_{i=1}^{M} W_{i} B_{i}$. This variable describes the mass observed in the interval $[a, b]$ when the null hypothesis is satisfied. When $M$ is large enough, the central limit theorem can be applied and leads to approximate the law of $D$ with a Normal law $\mathcal{N}\left(M p_{[a, b]} \lambda, M p \lambda^{2} *\left(2-p_{[a, b]}\right)\right)$. It follows that for all $\alpha$, we can approximate the probability $\mathbb{P}\left[D_{[a, b]}>\alpha\right]$ by

$$
F(\alpha):=\int_{\alpha}^{+\infty} g_{\mu, \sigma}(x) d x,
$$

with $\mu=M p_{[a, b]} \lambda$ and $\sigma=M p \lambda^{2} *\left(2-p_{[a, b]}\right)$. In consequence, the interval $[a, b]$ is said to be meaningful if it contains a mass $H_{[a, b]}$ such that

$$
F\left[H_{[a, b]}\right]<\frac{2}{N(N-1)} .
$$

The coefficient $\frac{N(N-1)}{2}$, corresponding to the number of intervals in the histogram $H^{9}$ is introduced to control the number of false alarms [13], [12]. Indeed, thresholding at $\frac{2}{N(N-1)}$ permits to ensure that the expected number of detected modes under the null hypothesis is smaller than one.

Intervals satisfying this test are selected. Then, a maximality criterion is applied, permitting to keep only intervals which neither contain, nor are contained in more meaningful intervals (intervals with a smaller $F\left[H_{[a, b]}\right]$ value). For each of the

\footnotetext{
${ }^{8}$ Recall that the density of the exponential distribution with parameter $\lambda$ is $f(x)=\frac{1}{\lambda} \exp (-x / \lambda)$.

${ }^{9}$ An interval, in this context, refers to any set of consecutive bins of $H$.
} 
maximal intervals, we apply the final procedure described in Algorithm 1 and for each mode selected, we average the contributing pixels to obtain an illuminant estimation.

\section{EXPERIMENTS}

In this section, we investigate the performance of our illuminant estimation algorithm. Our method is first evaluated and confronted with standard or state-of-the-art approaches on scenes containing a single illuminant. In order to assess the quality of the results, the comparison is performed on three different databases, namely the Colorchecker dataset [26], the Grey-Ball dataset [10] and the SFU laboratory dataset [3]. In the second part of this section, we test the ability of our method to deal with multi-illuminant situations.

\section{A. Colorspaces and error statistics}

The standard way to compare illuminant estimation algorithms is to measure angular errors between the estimated illuminants and the groundtruth illuminants provided with the databases (see Section V-B). Before describing more precisely the experimental protocol used in our experiments, it is important to specify which color spaces are used for computing these errors and why.

A great deal of illuminant estimation algorithms work directly in the RGB colorspace of the camera. Now, the colorspace of a camera depends on the sensitivity functions of its sensors. As a consequence, errors computed for two different cameras are not directly comparable, since the respective color components may have very different dynamic ranges for instance. This is the case for the Colorchecker database, which contain images taken with two different cameras, a Canon 5D and a Canon 1D. We believe that computing error statistics in a universal space is the only way to obtain error measures between illuminant estimations that are consistent from one camera to the other, and from one database to the other.

For all of these reasons, whenever possible, we provide the errors statistics of our algorithm both in the RGB space of the camera and in the standard colorspace sRGB 10 Since our approach provides an estimated illuminant in the standard chromaticity space uv, we convert the obtained estimation both to the camera colorspace and to sRGB. This permits to compute error statistics in each of these colorspaces.

\section{B. Single illuminant estimation}

1) Protocol: For the single illuminant evaluation, we follow an experimental protocol similar to the one described in [28]. Each image used in these experiments is provided with a measure $\mathbf{L}^{\mathbf{g}}$ of the light source illuminating the scene. Following [29], the angular error between this groundtruth and the estimated illuminant $L^{e}$ is defined as

$$
E_{a}\left(\mathbf{L}^{\mathbf{e}}, \mathbf{L}^{\mathbf{g}}\right)=\cos ^{-1}\left(\frac{\mathbf{L}^{\mathbf{e}} \cdot \mathbf{L}^{\mathbf{g}}}{\left\|\mathbf{L}^{\mathbf{e}}\right\| \cdot\left\|\mathbf{L}^{\mathbf{g}}\right\|}\right) .
$$

This error can be seen as an angle in a RGB space between two "grey" axes defined by the illuminants $\mathbf{L}^{\mathbf{e}}$ and $\mathbf{L}^{\mathrm{g}}$. Observe

\footnotetext{
${ }^{10} \mathrm{We}$ refer here to the linear sRGB colorspace (primaries and white point) We do not apply any gamma correction.
}

that the illuminant intensity is not involved in this error computation.

We compute several error statistics for each database. As noticed by the authors of [28], the error distribution is not symmetric and therefore, the mean is weakly informative. The median and the trimean measure are more meaningful statistics in practice.

2) Databases: We now describe the three databases used for the single illuminant comparison.

a) Colorchecker dataset: The Colorchecker dataset, provided by Gehler et al. [26], is composed of 568 images, captured with two different cameras (a Canon EOS-1D and a Canon EOS-5D). As a consequence, as said before, results computed in the camera RGB space, averaged in different spaces, should be taken cautiously. The database is composed of indoor and outdoor scenes, corresponding to a large variety of illuminants. These groundtruth illuminants are provided as RGB triplets in the respective colorspaces of the cameras. The two cameras used to create the dataset being known, we can easily convert these triplets in the XYZ colorspace 11 and visualize their projections on the CIE $1960 u v$ chromaticity diagram (see Figure 1).

Gehler et al. [26] provide the database in both RAW and TIFF versions. The TIFF version was found [49] to be subject to non-linear corrections such as gamma-correction, clipping and demosaicing. Our experiments therefore rely on the RAW versions.

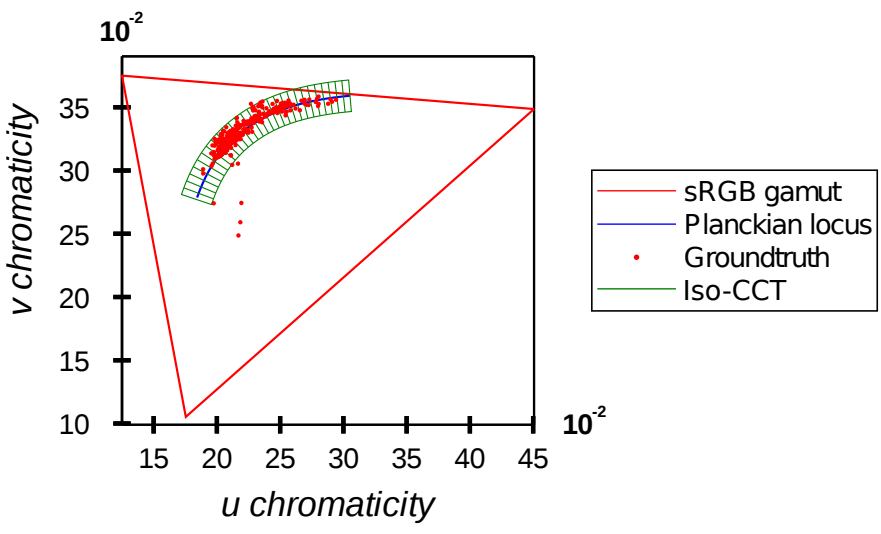

Fig. 1. Colorchecker illuminants groundtruth in the CIE $1960 u v$ diagram. The red triangular region represents the set of possible colors in SRGB and the blue line represents the Planckian locus (the chromaticities of the Planckian illuminants). The green region around the Planckian locus correspond to a distance threshold $\delta=0.0125$ and the green lines are iso-CCT lines. Each of the 568 red points corresponds to the $u v$ coordinates of an illuminant of the Colorchecker database. Observe that almost all the chromaticities of these illuminants lie in the chosen vicinity of the Planckian locus.

b) Grey-Ball dataset: The Grey-Ball dataset [10] comes with 11,346 JPEG images extracted from videos. As recommended by [28], the gamma correction has been removed in order to work with linear images. The output RGB system of the camera (Sony VX-200) being unknown, we assume a sRGB space for these images. For each image, a grey-ball is present in the visual field of the camera. The creators of the database use the pixels belonging to the grey-ball in order

\footnotetext{
${ }^{11}$ The conversion matrices are documented in dcraw [11].
} 
TABLE I

FIRST TABLE SHOWS PERFORMANCES ON THE COLORCHECKER DATASET [49] IN THE CAMERA RGB SPACE AND SECOND TABLE SHOWS RESULTS IN THE SRGB COLORSPACE. RESULTS ARE COMPARED TO LEARNING-FREE METHODS ([40], [7], [3], [50]) AND METHODS INVOLVING A TRAINING PHASE ([25], [27], [8]). OBSERVE THAT OUR ALGORITHM PROVIDES COMPARABLE PERFORMANCES WITH MORE COMPLEX LEARNING-BASED ALGORITHMS.

\begin{tabular}{|l|c|c|c|c|c|}
\hline Method & Mean & Median & Trimean & Best-25\% & Worst-25\% \\
\hline \hline White-Patch [40] & $7.5^{\circ}$ & $5.7^{\circ}$ & $6.4^{\circ}$ & $1.5^{\circ}$ & $16.2^{\circ}$ \\
Grey-World [7] & $6.4^{\circ}$ & $6.3^{\circ}$ & $6.3^{\circ}$ & $2.3^{\circ}$ & $10.6^{\circ}$ \\
general Grey-World [3] & $4.7^{\circ}$ & $3.5^{\circ}$ & $3.8^{\circ}$ & $1.0^{\circ}$ & $10.2^{\circ}$ \\
1st - order Grey-Edge [50] & $5.3^{\circ}$ & $4.5^{\circ}$ & $4.7^{\circ}$ & $1.8^{\circ}$ & $10.2^{\circ}$ \\
\hline Pixel-based Gamut Mapping [25] & $4.2^{\circ}$ & $2.3^{\circ}$ & $2.9^{\circ}$ & $0.5^{\circ}$ & $10.8^{\circ}$ \\
Edge-based Gamut Mapping [27] & $6.5^{\circ}$ & $5.0^{\circ}$ & $5.4^{\circ}$ & $1.9^{\circ}$ & $13.6^{\circ}$ \\
ML (category-wise prior) [8] & $3.7^{\circ}$ & $3.0^{\circ}$ & $3.1^{\circ}$ & $1.0^{\circ}$ & $7.6^{\circ}$ \\
\hline Proposed method $\delta=0.02$ & $4.1^{\circ}$ & $2.7^{\circ}$ & $3.1^{\circ}$ & $0.8^{\circ}$ & $9.6^{\circ}$ \\
Proposed method (fixed parameters) & $4.5^{\circ}$ & $3.1^{\circ}$ & $3.5^{\circ}$ & $0.8^{\circ}$ & $10.8^{\circ}$ \\
\hline
\end{tabular}

\begin{tabular}{|l|c|c|c|c|c|}
\hline Method & Mean & Median & Trimean & Best-25\% & Worst-25\% \\
\hline \hline White-Patch [40] & $7.9^{\circ}$ & $5.6^{\circ}$ & $6.2^{\circ}$ & $1.7^{\circ}$ & $18.0^{\circ}$ \\
Grey-World [7] & $6.9^{\circ}$ & $5.0^{\circ}$ & $5.5^{\circ}$ & $1.3^{\circ}$ & $15.6^{\circ}$ \\
general Grey-World [3] & $5.2^{\circ}$ & $3.2^{\circ}$ & $3.7^{\circ}$ & $0.7^{\circ}$ & $12.9^{\circ}$ \\
1st - order Grey-Edge [50] & $6.0^{\circ}$ & $3.9^{\circ}$ & $4.6^{\circ}$ & $1.2^{\circ}$ & $14.0^{\circ}$ \\
\hline Proposed method $\delta=0.02$ & $5.0^{\circ}$ & $2.9^{\circ}$ & $3.4^{\circ}$ & $0.7^{\circ}$ & $13.0^{\circ}$ \\
Proposed method (fixed parameters) & $5.2^{\circ}$ & $3.0^{\circ}$ & $3.5^{\circ}$ & $0.7^{\circ}$ & $13.4^{\circ}$ \\
\hline
\end{tabular}

to deduce the groundtruth illuminants and to provide these illuminants as RGB triplets 12 As shown on Figure 2, this dataset covers a large variety of illuminants.

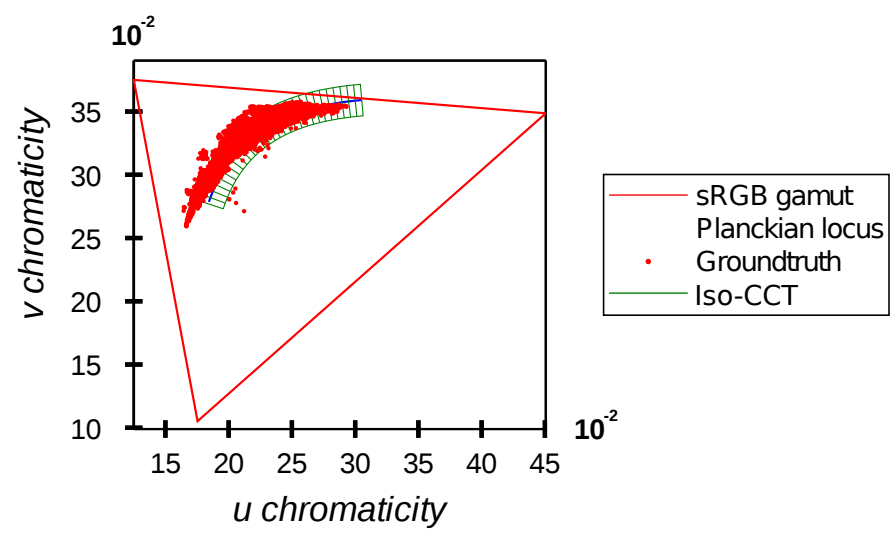

Fig. 2. Grey-Ball groundtruth illuminants projected in the CIE 1960 uv chromaticity diagram.

c) SFU laboratory dataset: This database [3] contains 321 images from 31 different scenes, each one being illuminated by 11 light sources. Scenes generally contain only few objects, the range of colors in images is thus limited. This database is therefore a challenging case for color constancy algorithms.

For each image in the database, the authors provide the spectra of the groundtruth illuminants and the corresponding triplets in the colorspace of the camera. When projecting these spectra onto the $x y$ diagram, as shown in the left part of Figure 3, we observe that all of these illuminants are in the vicinity of the Planckian locus. One image in the database contains a colorchecker: we use this image and the knowledge of the corresponding illuminant to estimate the conversion

\footnotetext{
${ }^{12}$ Pixels belonging to the grey-ball are discarded from the comparative experiments.
}

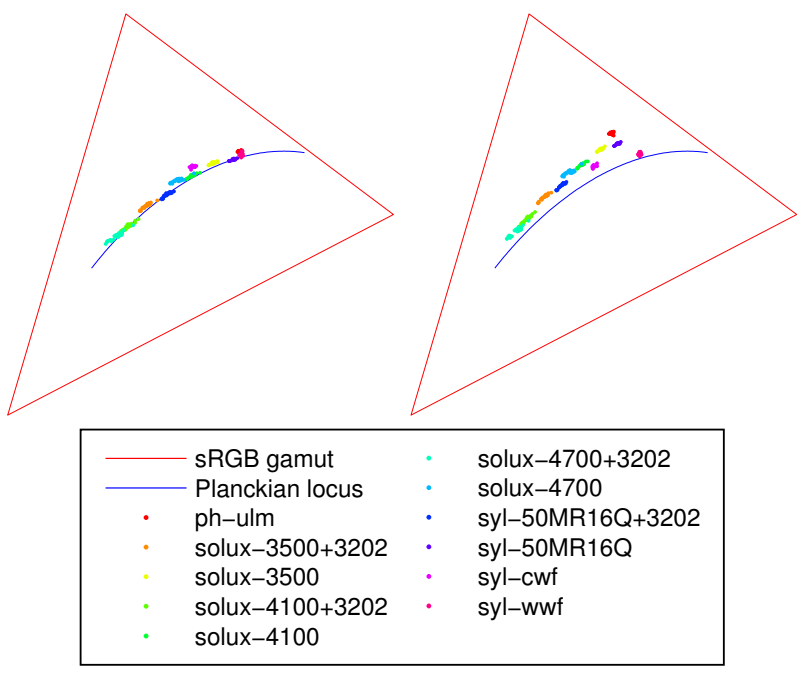

Fig. 3. Groundtruth illuminants in the xy diagram for the SFU LAB database. On the left, the direct projection of illuminant spectra in the xy diagram. On the right, projections of the illuminant triplets provided with the database after their conversion to XYZ. The difference between the two projections is explained by an imperfect compatibility between the camera colorspace and XYZ.

matrix between the camera colorspace and XYZ ${ }^{13}$. This permits to project onto the $x y$ diagram the illuminant triplets provided along with the illuminant spectra, as shown on the right part of Figure 3 . Surprisingly, the projections obtained with these triplets differ noticeably from the ones obtained with the spectra, which means that the sensitivity functions of the camera used to create the database do not provide a perfect matching between the camera colorspace and XYZ. In practice, this problem should be less significant with recent cameras, which now offer a good compatibility with standard colorspaces.

\footnotetext{
${ }^{13}$ Note that in this case, the conversion matrix could also be estimated thanks to the camera sensitivity functions. In practice, these sensitivity functions are rarely known, while it is always possible to take a picture of a colorchecker under a known illuminant.
} 
TABLE II

PERFormances on the LiNEAR SFU GREY-BALl DATABASE, IN THE CAMERA COlORSPACE. ON THIS DATASET, IT CAN BE OBSERVED THAT DESPITE THE LARGE VARIABILITY OF ILLUMINANTS (SEE FIGURE2, THE PROPOSED ALGORITHM STILL PROVIDES STATE OF THE ART RESULTS.

\begin{tabular}{|l|c|c|c|c|c|}
\hline Method & Mean & Median & Trimean & Best-25\% & Worst-25\% \\
\hline \hline White-Patch [40] & $12.7^{\circ}$ & $10.5^{\circ}$ & $11.3^{\circ}$ & $2.5^{\circ}$ & $26.2^{\circ}$ \\
Grey-World [7] & $13.0^{\circ}$ & $11.0^{\circ}$ & $11.5^{\circ}$ & $3.1^{\circ}$ & $26.0^{\circ}$ \\
general Grey-World [3] & $11.6^{\circ}$ & $9.7^{\circ}$ & $10.2^{\circ}$ & $3.4^{\circ}$ & $22.7^{\circ}$ \\
1st - order Grey-Edge [50] & $10.6^{\circ}$ & $8.8^{\circ}$ & $9.2^{\circ}$ & $3.0^{\circ}$ & $21.1^{\circ}$ \\
\hline Pixel-based Gamut Mapping [25] & $11.8^{\circ}$ & $8.9^{\circ}$ & $10.0^{\circ}$ & $2.8^{\circ}$ & $24.9^{\circ}$ \\
Edge-based Gamut Mapping [27] & $12.8^{\circ}$ & $10.9^{\circ}$ & $11.4^{\circ}$ & $3.6^{\circ}$ & $25.0^{\circ}$ \\
ML [8] & $10.3^{\circ}$ & $8.9^{\circ}$ & $9.2^{\circ}$ & $2.8^{\circ}$ & $20.3^{\circ}$ \\
\hline Our method $(\delta=0.0075)$ & $10.5^{\circ}$ & $8.2^{\circ}$ & $8.9^{\circ}$ & $2.2^{\circ}$ & $22.5^{\circ}$ \\
Our method (fixed parameters) & $11.1^{\circ}$ & $9.1^{\circ}$ & $9.5^{\circ}$ & $2.6^{\circ}$ & $23.0^{\circ}$ \\
\hline
\end{tabular}

3) Comparative evaluation: Tables $\mathrm{I}$ to $\mathrm{V}$ show the results obtained with the three different databases. In each table, the results of our approach are provided with two different sets of parameters: a set of fixed parameters, identical for all experiments and provided in Algorithm 1 and a set where the threshold $\delta$ is optimized for the database.

a) Colorchecker: For the Colorchecker dataset, results are provided both in the colorspace of the camera and in the sRGB colorspace (see Table I).

Error statistics from state of the art approaches in the camera chromaticity space are reproduced from [28]. As explained before, error statictics in the camera colorspace should be taken with caution, since the database is composed of two different cameras. It also appears that the results provided in the literature on this database were computed from RAW images without removing the black level of the camera (which is different for these two cameras), which also introduces bias in the results (for fair comparison, we did the same for our algorithm).

Error statistics of common learning-free methods in SRGB are computed by running these algorithms directly on images converted to sRGB, after removing the camera black level (129 for the Canon 5D and 0 for the Canon 1D). Interestingly, there is a reversal of role in the relative performances of the GreyWorld and White-Patch algorithms when comparing their error statistics in sRGB and RGB. This confirms the importance of the colorspace chosen to run the different algorithms and to compute the error statistics.

Observe that in both colorspaces, our approach shows better results than standard, learning-free methods. We also see from this table that our approach provides comparable performances with more complex learning-based algorithms, while keeping a very reasonable computational cost.

b) Grey-Ball: On the Grey-Ball database (see Table II], our approach also shows better results than standard illuminant estimation methods. On this database, the results of our approach are even slightly better than the more complex, state of the art learning-based methods if the parameter $\delta$ is well chosen. Observe also that the algorithm is quite stable, providing good results both in the Colorchecker and in the Grey-Ball databases with the same set of parameters.

c) SFU Lab: For the SFU Lab database, results are also provided both in SRGB and in the camera colorspace. Table 【II first shows the statistical errors on the entire database. As explained before, these results should be interpreted with
TABLE IV

Performances on the SFU lab Database With RESPECT TO THE ILLUMINANT.

\begin{tabular}{|l|c|c|c|}
\hline Illuminant & Mean & Median & Worst-25\%( $\mu)$ \\
\hline \hline ph-ulm & $8.5^{\circ}$ & $4.6^{\circ}$ & $21.5^{\circ}$ \\
solux-3500+3202 & $5.3^{\circ}$ & $1.9^{\circ}$ & $16.6^{\circ}$ \\
solux-3500 & $7.2^{\circ}$ & $2.7^{\circ}$ & $22.6^{\circ}$ \\
solux-4100+3202 & $4.8^{\circ}$ & $1.6^{\circ}$ & $15.7^{\circ}$ \\
solux-4100 & $6.5^{\circ}$ & $2.9^{\circ}$ & $20.3^{\circ}$ \\
solux-4700+3202 & $4.5^{\circ}$ & $1.5^{\circ}$ & $15.2^{\circ}$ \\
solux-4700 & $7.1^{\circ}$ & $2.7^{\circ}$ & $21.1^{\circ}$ \\
syl-50MR16Q+3202 & $5.9^{\circ}$ & $2.1^{\circ}$ & $18.7^{\circ}$ \\
syl-50MR16Q & $7.3^{\circ}$ & $2.9^{\circ}$ & $22.4^{\circ}$ \\
syl-cwf & $6.9^{\circ}$ & $3.5^{\circ}$ & $20.7^{\circ}$ \\
syl-wwf & $5.9^{\circ}$ & $4.6^{\circ}$ & $14.6^{\circ}$ \\
\hline
\end{tabular}

caution because the RGB color space of the camera used in this database is not perfectly compatible with standard colorspaces, which is a prerequisite of our algorithm. Despite this drawback, the performance of our approach on this database is relatively close to those of the most recent state of the art algorithms. Looking at the median and trimean columns, we see that our approach yields slightly lower performances than the best learning-based approaches. On the Best $25 \%$ column, our results are slightly better. This can be explained by a large variance of the error distribution on this database. When a scene satisfies the algorithm assumptions, that is if there is a grey surface in the scene, or a set of very light grey dots, the algorithm yields excellent results. Otherwise, the results are very poor, as shown in the Worst$25 \%$ column. This is illustrated by Table V, which shows the errors statistics depending on the kind of observed scene. As might be expected, the scenes containing achromatic surfaces, shown on the top of Figure 4 produce excellent results. On the contrary, scenes without achromatic surfaces are subject to very large estimation errors. Table IV] shows that error statistics are much less dependent on the type of illuminant used than on the type of scene.

4) Parameter selection: We comment below the influence of the different parameters of the algorithm. The parameters $\delta$, $T_{\min }$ and $T_{\max }$ delimit the set of colors considered as being potentially achromatic (we refer to this set as the illuminant gamut). From our experiments, it appears that the result of our algorithm is not sensitive to small modifications of $T_{\min }$ and $T_{\max }$. The choice of $\delta$ has a stronger impact on the results, but in practice the choice of $\delta=0.0125$ has led to 


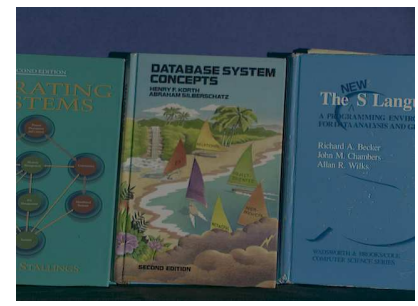

(a) Books

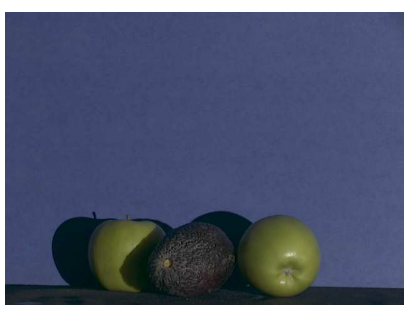

(e) Apples

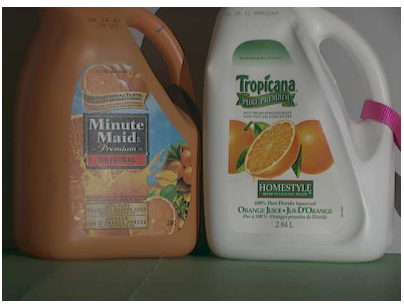

(b) Plastic

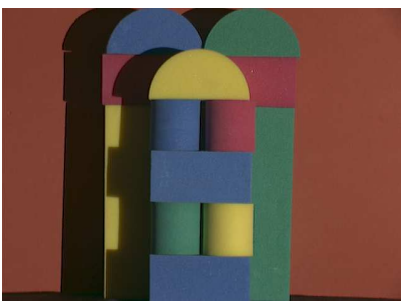

(f) Blocks

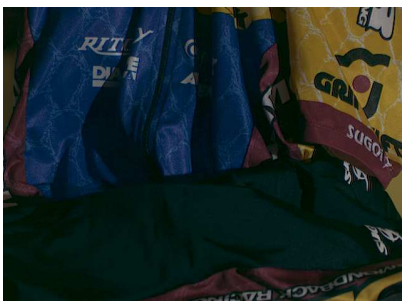

(c) Jersey

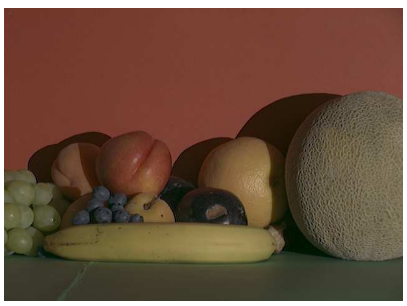

(g) Fruits

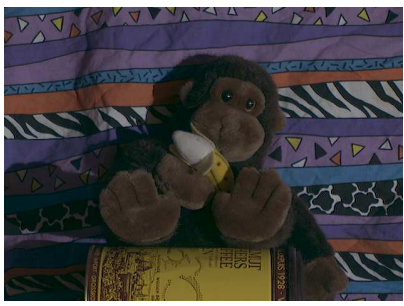

(d) Monkey

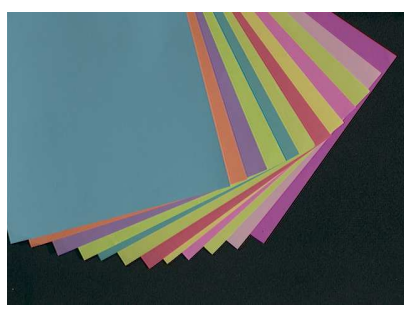

(h) Papers

Fig. 4. Examples of scenes for which the algorithm hypotheses are satisfied (top) or not (bottom).

TABLE III

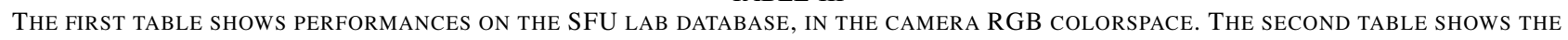
COMPARATIVE RESULTS IN THE UNIVERSAL SRGB COLORSPACE.

\begin{tabular}{|l|c|c|c|c|c|}
\hline Method & Mean & Median & Trimean & Best-25\% & Worst-25\% \\
\hline \hline White-Patch [40] & $9.1^{\circ}$ & $6.5^{\circ}$ & $7.5^{\circ}$ & $1.8^{\circ}$ & $21.1^{\circ}$ \\
Grey-World [7] & $9.8^{\circ}$ & $7.0^{\circ}$ & $7.6^{\circ}$ & $0.9^{\circ}$ & $23.6^{\circ}$ \\
general Grey-World [3] & $5.4^{\circ}$ & $3.3^{\circ}$ & $3.8^{\circ}$ & $0.5^{\circ}$ & $13.8^{\circ}$ \\
1st - order Grey-Edge [50] & $5.6^{\circ}$ & $3.2^{\circ}$ & $3.7^{\circ}$ & $1.1^{\circ}$ & $14.1^{\circ}$ \\
\hline GSI [54] & - & $3.9^{\circ}$ & - & - & - \\
Cluster [55] & - & $3.6^{\circ}$ & - & - & - \\
\hline Pixel-based Gamut Mapping [25] & $3.7^{\circ}$ & $2.3^{\circ}$ & $2.5^{\circ}$ & $0.5^{\circ}$ & $9.4^{\circ}$ \\
Edge-based Gamut Mapping [27] & $3.9^{\circ}$ & $2.3^{\circ}$ & $2.7^{\circ}$ & $0.5^{\circ}$ & $10.0^{\circ}$ \\
\hline Our method ( $\delta=0.015)$ & $6.4^{\circ}$ & $2.4^{\circ}$ & $3.6^{\circ}$ & $0.3^{\circ}$ & $19.3^{\circ}$ \\
Our method (Fixed parameters) & $6.5^{\circ}$ & $2.7^{\circ}$ & $4.0^{\circ}$ & $0.3^{\circ}$ & $19.0^{\circ}$ \\
\hline
\end{tabular}

\begin{tabular}{|l|c|c|c|c|c|}
\hline Method & Mean & Median & Trimean & Best-25\% & Worst-25\% \\
\hline \hline White-Patch [40] & $6.3^{\circ}$ & $4.9^{\circ}$ & $5.2^{\circ}$ & $1.4^{\circ}$ & $13.8^{\circ}$ \\
Grey-World [7] & $10.9^{\circ}$ & $8.0^{\circ}$ & $8.7^{\circ}$ & $1.3^{\circ}$ & $26.2^{\circ}$ \\
general Grey-World [3] & $4.6^{\circ}$ & $3.2^{\circ}$ & $3.4^{\circ}$ & $0.5^{\circ}$ & $10.9^{\circ}$ \\
1st - order Grey-Edge [50] & $5.8^{\circ}$ & $3.4^{\circ}$ & $4.0^{\circ}$ & $1.1^{\circ}$ & $14.6^{\circ}$ \\
\hline Our method $(\delta=0.015)$ & $5.3^{\circ}$ & $1.9^{\circ}$ & $2.5^{\circ}$ & $0.3^{\circ}$ & $16.4^{\circ}$ \\
Our method (Fixed parameters) & $6.2^{\circ}$ & $2.6^{\circ}$ & $3.7^{\circ}$ & $0.4^{\circ}$ & $17.7^{\circ}$ \\
\hline
\end{tabular}

TABLE V

PERFormances on the SFU LAB DATABASE With RESPECT TO THE TYPE OF SCENE.

\begin{tabular}{|l|c|c|c|}
\hline Scene & Mean & Median & Worst-25\% $(\mu)$ \\
\hline \hline Apples & $21.4^{\circ}$ & $22.5^{\circ}$ & $29.7^{\circ}$ \\
Blocks & $27.4^{\circ}$ & $29.3^{\circ}$ & $31.2^{\circ}$ \\
Fruit & $20.0^{\circ}$ & $20.4^{\circ}$ & $25.7^{\circ}$ \\
Paper & $14.0^{\circ}$ & $12.5^{\circ}$ & $20.2^{\circ}$ \\
\hline Books & $4.2^{\circ}$ & $3.3^{\circ}$ & $7.3^{\circ}$ \\
Jersey & $1.7^{\circ}$ & $1.6^{\circ}$ & $2.5^{\circ}$ \\
Monkey & $0.8^{\circ}$ & $0.8^{\circ}$ & $1.7^{\circ}$ \\
Plastic & $2.0^{\circ}$ & $1.0^{\circ}$ & $4.9^{\circ}$ \\
\hline
\end{tabular}

good performances for all the tested datasets. Observe that the algorithm is prone to fail on an image if the groundtruth illuminant is located far outside of the illuminant gamut. A possible improvement for these specific cases would consist in replacing the hard threshold $\delta$ by a soft one. Let us also mention that weighting the contribution of the pixels in the histogram with a power of the luminance is quite important in practice, although the performances of the algorithm are not radically affected by the choice of this power.

\section{Multi illuminant estimation}

In this section, we evaluate the ability of our approach to deal with scenes lit by multiple light sources. As described in Section IV] the mode selection occurring at the end of our algorithm can be extended to detect several modes in the temperature histogram $H$. In practice, we assume that each of these detected modes has been generated by a light source.

Observe that removing the uniqueness assumption for the illuminant is potentially dangerous. Indeed, the inherent ambiguity of the illuminant estimation problem (an observed color may correspond to a neutral surface seen under a colored illuminant as well as a colored surface seen under a canonical 
illuminant) increases when the number of light sources is unknown.

Evaluating the algorithm in this multi-illuminant framework raises two questions. First, when only one light source is used in the scene, to what extent the multiple mode detection can deteriorate performances? Second, when several illuminants are indeed involved in the scene, how the results are improved by detecting these secondary modes ? We suggest an experiment for each of these questions.

1) Measuring errors between two sets of illuminants: Before describing these experiments, let us detail how we measure the error between an arbitrary number of illuminants and estimations. For this task, we make use of the EMD distance introduced by Rubner et al. in [46]. Let $N^{g}$ be the number of groundtruth illuminants present in the scene and let $N^{e}$ be the number of estimated illuminants. We attribute a mass $1 / N^{g}$ to each groundtruth illuminant and a mass $1 / N^{g}$ to each one of our estimations. We then compute the EMD distance between the two sets of illuminants, the cost function between two illuminants being defined as their euclidean distance in the $r g$ chromaticity diagram.

2) Multi-illuminant case: There is no standard dataset for the evaluation of multi-illuminant detection algorithms. In order to evaluate our algorithm, we follow the protocol previously proposed by Gijsenij et al. in [31]. We create a synthetic dataset from the Colorchecker database. Each image is first corrected using the provided groundtruth illuminant and the Von Kries model. Then, for each corrected image, two illuminants $L^{1}$ and $L^{2}$ are randomly selected ${ }^{14}$ in the set of groundtruth illuminants provided with the database. The corrected image is then illuminated by these two light sources, a mask defining the local proportions of each illuminant, as illustrated by Figure 5 . Several masks are possible (vertical, horizontal or diagonal). In order to smooth the transition between illuminants, Gaussian filtering with random standard deviation (between 1 and 5) is used to blur the binary masks.

3) Single illuminant case: For the single illuminant case, we reproduce partially the experiment on the Colorchecker dataset [49] described in Section V-B, except that we allow our algorithm to detect several meaningful modes. The experiment illustrates the behavior of our algorithm when the a priori on the number of illuminant is removed. The performance of the algorithm can only decrease in this framework, the goal of the experiment is to measure to which extent.

4) Results: Table VI shows the performance of our algorithm on the set of images generated using two light sources, as described in V-C2 Our algorithm is tested twice on this dataset. In the first experiment, we deliberately keep only one mode (the most meaningful one) in the histogram. In the second experiment, we release the uniqueness assumption and we keep all the maximal meaningful modes. In this multiilluminant framework, authorizing the detection of multiple modes permits to increase quite significantly the detection accuracy.

\footnotetext{
${ }^{14}$ We check that these two illuminants are not too close in practice, and discard the pairs such that $\operatorname{EMD}\left(L^{1}, L^{2}\right)>1.11$. This threshold roughly corresponds to the one used in [31].
}

Table VII shows the performance loss resulting from the relaxation of the uniqueness assumption when applying our algorithm on the original images of the Colorchecker dataset. The first line of the table shows the error statistics when the number of illuminants to be detected is not imposed at all, and the second line shows the performance of the single mode detection. Observe that the performance loss between both configurations is very reasonable. We insist on the fact that to the best of our knowledge, no algorithm had been proposed in the literature to estimate at the same time the number of illuminant present in the scene and the color of these illuminants.

\section{CONCLUSION}

In this paper, a new procedure for the estimation of single or multiple illuminants in color images has been proposed. A novel and simple voting scheme has been introduced and combined with physical constraints, in order to select the most probable illuminants in a chromaticity space. It has been shown that this algorithm permits to achieve state of the art performances on different challenging databases without requiring any learning step. The ability of the whole procedure to deal with situations where the number of light sources is unknown has also been demonstrated. To the best of our knowledge, such ability was not reported before in the literature. The proposed procedure works globally on images, even in the multi-illuminant case. This strongly differs from several recent approaches working locally on image patches or superpixels [5], [31]. These methods enable local color corrections, but cannot automatically detect the number of light sources. As a future work, we intend to explore connexions and tradeoffs between these local procedures and our approach.

\section{REFERENCES}

[1] Kobus Barnard, Vlad Cardei, and Brian Funt. A comparison of computational color constancy algorithms. i: Methodology and experiments with synthesized data. Image Processing, IEEE Transactions on, 11(9):972984, 2002.

[2] Kobus Barnard, Graham Finlayson, and Brian Funt. Color constancy for scenes with varying illumination. Computer vision and image understanding, 65(2):311-321, 1997.

[3] Kobus Barnard, Lindsay Martin, Adam Coath, and Brian Funt. A comparison of computational color constancy algorithms. IEEE Trans. Image Process., 11:2002, 2002.

[4] Simone Bianco, Gianluigi Ciocca, Claudio Cusano, and Raimondo Schettini. Improving color constancy using indoor-outdoor image classification. Image Processing, IEEE Transactions on, 17(12):23812392, 2008.

[5] Michael Bleier, Christian Riess, Shida Beigpour, Eva Eibenberger, Elli Angelopoulou, Tobias Trger, and Andr Kaup. Color constancy and non-uniform illumination: Can existing algorithms work? In ICCV Workshops, 2011.

[6] Ivaylo Boyadzhiev, Kavita Bala, Sylvain Paris, and Frédo Durand. Userguided white balance for mixed lighting conditions. ACM Trans. Graph., 31(6):200:1-200:10, November 2012.

[7] G Buchsbaum. A spatial processor model for object colour perception. J. Franklin Inst., 310(1):1-26, 1980.

[8] Ayan Chakrabarti, Keigo Hirakawa, and Todd Zickler. Color constancy with spatio-spectral statistics. Pattern Analysis and Machine Intelligence, IEEE Transactions on, 34(8):1509-1519, 2012.

[9] Feng-Ju Chang, Soo-Chang Pei, and Wei-Lun Chao. Color constancy by chromaticity neutralization. JOSA A, 29(10):2217-2225, 2012.

[10] Florian Ciurea and Brian Funt. A large image database for color constancy research, 2003.

[11] Dave Coffin. Decoding raw digital photos in linux, 2013. 


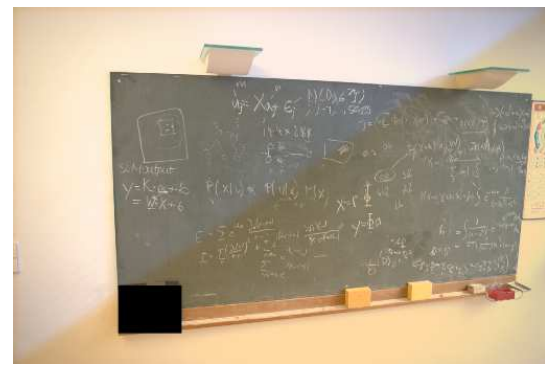

(a) Image sample

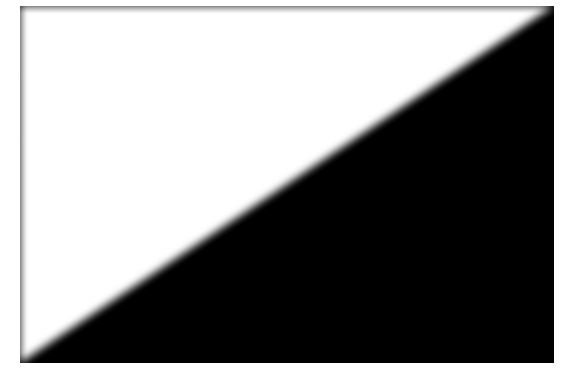

(b) Mask

Fig. 5. Left: exemple of an image created for estimating multiples light sources. Right: mask used to define the spatial proportion of each illuminant.

TABLE VI

RESUlts on IMAGES GENERATED USING TWO LIGHT SOURCES (SEE SECTIONV-C2). FiRST LINE: THE ALGORITHM ASSUMES ONE SINGLE LIGHT SOURCE. SECOND LINE: THE UNIQUENESS ASSUMPTION IS REMOVED. $k=3$, delta $=0.0150, \mathrm{Tm}=2000, \mathrm{TM}=20000$, bins $=300$

\begin{tabular}{|l|c|c|c|c|c|c|c|}
\hline Method & Crit. & Mean & Med & Trimean & Best-25\% & Worst-25\% & Max. \\
\hline \hline Single mode & 5.8 & 6.2 & 5.3 & 5.6 & 1.3 & 12.6 & 26.1 \\
Multiple modes & 3.7 & 4.5 & 2.9 & 3.4 & 0.3 & 11.0 & 20.3 \\
\hline
\end{tabular}

TABLE VII

RESULTS ON THE COLORCHECKER DATASET WHEN NO PRIOR INFORMATION ON THE NUMBER OF ILLUMINANTS IS PROVIDED. $k=3$, delta $=0.0150, T m=2000, T M=20000$, bins $=100$

\begin{tabular}{|l|c|c|c|c|c|c|c|}
\hline Method & Crit. & Mean & Med & Trimean & Best-25\% & Worst-25\% & Max. \\
\hline \hline Multiple modes & 3.9 & 4.7 & 3.1 & 3.6 & 0.6 & 11.2 & 23.1 \\
Single mode & 2.8 & 3.7 & 2.0 & 2.4 & 0.5 & 9.6 & 25.6 \\
\hline
\end{tabular}

[12] A. Desolneux, L. Moisan, and J.-M. Morel. A grouping principle and four applications. PAMI, 25(4):508-513, 2003.

[13] A. Desolneux, L. Moisan, and J. M. Morel. From Gestalt Theory to Image Analysis, volume 34. Springer-Verlag, 2008.

[14] Marc Ebner. A parallel algorithm for color constancy. J. Parallel Distrib. Comput., 64(1):79-88, January 2004.

[15] Marc Ebner. Color constancy, volume 6. Wiley, 2007.

[16] Marc Ebner. Estimating the color of the illuminant using anisotropic diffusion. In Walter G. Kropatsch, Martin Kampel, and Allan Hanbury, editors, Computer Analysis of Images and Patterns, 12th International Conference, CAIP 2007, Vienna, Austria, August 27-29, 2007, Proceedings, volume 4673 of Lecture Notes in Computer Science, pages 441449. Springer, 2007.

[17] G. D. Finlayson and S. D. Hordley. Gamut constrained illuminant estimation. Int. J. Comput. Vision, 67:2006, 2006.

[18] Graham D. Finlayson, Mark S. Drew, and Brian V. Funt. Color constancy: Generalized diagonal transforms suffice. J. Opt. Soc. Am. A, 11:3011-3020, 1994.

[19] Graham D. Finlayson, Brian V. Funt, and Kobus Barnard. Color constancy under varying illumination. In ICCV, pages 720-725, 1995.

[20] Graham D Finlayson and Steven D Hordley. Color constancy at a pixel. JOSA A, 18(2):253-264, 2001.

[21] Graham D. Finlayson and Steven D. Hordley. Color constancy at a pixel. J. Opt. Soc. Am. A, 18(2):253-264, Feb 2001.

[22] Graham D. Finlayson, Steven D. Hordley, and Paul M. Hubel. Color by correlation: A simple, unifying framework for color constancy. Pattern Analysis and Machine Intelligence, IEEE Transactions on, 23(11):1209_ 1221, 2001.

[23] Graham D. Finlayson and Gerald Schaefer. Solving for colour constancy using a constrained dichromatic reflection model. Int. J. Comput. Vision, 42(3):127-144, May 2001.

[24] Graham D. Finlayson and Elisabetta Trezzi. Shades of gray and colour constancy. In Color Imaging Conference, pages 37-41, 2004.

[25] D. A. Forsyth. A novel algorithm for color constancy. Int. J. Comput. Vision, 5(1):5-36, 1990.

[26] P. V. Gehler, C. Rother, A. Blake, T. Minka, and T. Sharp. Bayesian color constancy revisited. In Conference on Computer Vision and Pattern Recognition, pages 1-8, 062008.

[27] A. Gijsenij, T. Gevers, and J. van de Weijer. Generalized gamut mapping using image derivative structures for color constancy. Int. J. Comput. Vision, 86(2-3):127-139, 2010.

[28] A. Gijsenij, T. Gevers, and J. van de Weijer. Computational color constancy: Survey and experiments. IEEE Trans. Image Process., 20(9):2475-2489, 2011.

[29] A. Gijsenij, Th. Gevers, and M.P Lucassen. A perceptual analysis of distance measures for color constancy. Journal of the Optical Society of America A, 26(10):2243-2256, 2009.

[30] A. Gijsenij, Th. Gevers, and J. van de Weijer. Physics-based edge evaluation for improved color constancy. In IEEE Computer Society Conference on Computer Vision and Pattern Recognition 2009 (CVPR'09), pages 1-8, Miami Beach, Florida, USA, June 2009.

[31] A. Gijsenij, Rui Lu, and T. Gevers. Color constancy for multiple light sources. Image Processing, IEEE Transactions on, 21(2):697 -707, feb. 2012.

[32] Arjan Gijsenij and Theo Gevers. Color constancy using natural image statistics and scene semantics. IEEE Trans. Pattern Anal. Mach. Intell., 33(4):687-698, 2011.

[33] Javier Hernández-Andrés, Raymond L. Lee, and Javier Romero. Calculating correlated color temperatures across the entire gamut of daylight and skylight chromaticities. Appl. Opt., 38(27):5703-5709, Sep 1999.

[34] Eugene Hsu, Tom Mertens, Sylvain Paris, Shai Avidan, and Frédo Durand. Light mixture estimation for spatially varying white balance. ACM Trans. Graph., 27(3):70:1-70:7, August 2008.

[35] Yoshie Imai, Yu Kato, Hideki Kadoi, Takahiko Horiuchi, and Shoji Tominaga. Estimation of multiple illuminants based on specular highlight detection. In Proceedings of the Third international conference on Computational color imaging, CCIW'11, pages 85-98, Berlin, Heidelberg, 2011. Springer-Verlag.

[36] Deane B. Judd, David L. Macadam, Gütnter Wyszecki, H. W. Budde, H. R. Condit, S. T. Henderson, and J. L. Simonds. Spectral distribution of typical daylight as a function of correlated color temperature. J. Opt. Soc. Am., 54(8):1031-1036, Aug 1964.

[37] Rei Kawakami, Katsushi Ikeuchi, and Robby T. Tan. Consistent surface color for texturing large objects in outdoor scenes. In Proceedings of the Tenth IEEE International Conference on Computer Vision - Volume 2, ICCV '05, pages 1200-1207, Washington, DC, USA, 2005. IEEE Computer Society.

[38] Rei Kawakami, Robby T Tan, and Katsushi Ikeuchi. A robust frame- 
work to estimate surface color from changing illumination. In Asian Conference on Computer Vision (ACCV2004), 2004.

[39] Hyuk-Ju Kwon, Sung-Hak Lee, Tae-Wuk Bae, and Kyu-Ik Sohng. Compensation of de-saturation effect in $\{$ HDR $\}$ imaging using a real scene adaptation model. Journal of Visual Communication and Image Representation, 24(6):678 - 685, 2013.

[40] E.H. Land and John J. McCann. Lightness and retinex theory. J. Opt. Soc. Am., 61(1):1-11, Jan 1971.

[41] Bing Li, De Xu, Weihua Xiong, and Songhe Feng. Color constancy using achromatic surface. Color Research \& Application, 35(4):304312, 2010.

[42] Baptiste Mazin, Julie Delon, and Yann Gousseau. Illuminant estimation from projections on the planckian locus. In ECCV Workshops (2), pages 370-379, 2012.

[43] Irwin G. Priest. A proposed scale for use in specifying the chromaticity of incandescent illuminants and various phases of daylight. J. Opt. Soc. Am., 23(2):41-45, Feb 1933.

[44] Whitman Richards and Edward A Parks. Model for color conversion. JOSA, 61(7):971-976, 1971.

[45] Charles Rosenberg, Martial Hebert, and Sebastian Thrun. Color constancy using kl-divergence. In Computer Vision, 2001. ICCV 2001. Proceedings. Eighth IEEE International Conference on, volume 1, pages 239-246. IEEE, 2001.

[46] Y. Rubner, C. Tomasi, and L. J. Guibas. A metric for distributions with applications to image databases. In Proceedings of the 1998 IEEE International Conference on Computer Vision, pages 59-66, January 1998.

[47] Guillermo Sapiro. Color and illuminant voting. IEEE Trans. Pattern Anal. Mach. Intell., 21:1210-1215, 1999.

[48] Steven A Shafer. Using color to separate reflection components. Color Research \& Application, 10(4):210-218, 1985.

[49] Lilong Shi and Brian. Re-processed version of the gehler color constancy dataset of 568 images, 2010.

[50] J. Van De Weijer, T. Gevers, and A. Gijsenij. Edge-based color constancy. IEEE Trans. Image Process., 16(9):2207-2214, 2007.

[51] Joost Van De Weijer, Cordelia Schmid, and Jakob Verbeek. Using highlevel visual information for color constancy. In Computer Vision, 2007. ICCV 2007. IEEE 11th International Conference on, pages 1-8. IEEE, 2007.

[52] Javier Vazquez-Corral, Maria Vanrell, Ramon Baldrich, and Francesc Tous. Color constancy by category correlation. IEEE Transactions on Image Processing, 21(4):1997-2007, April 2012.

[53] Günther Wyszecki and W. S. Stiles. Color Science: Concepts and Methods, Quantitative Data and Formulae. Wiley-Interscience, 2 edition, August 2000.

[54] Weihua Xiong, Brian Funt, Lilong Shi, Sung-Su Kim, Byoung-Ho Kang, Sung-Duk Lee, and Chang-Yeong Kim. Automatic white balancing via gray surface identification. Color and Imaging Conference, 2007(1):143-146, 2007.

[55] Weihua Xiong, Jiangtao Kuang, and Xiaoyong Wang. Cluster based color constancy. Color and Imaging Conference, 2008(1):210-214, 2008 . 